\title{
Dream Recall Frequency Is Associated With Medial Prefrontal Cortex White-Matter Density
}

\author{
Raphael Vallat, Jean-Baptiste Eichenlaub, Alain Nicolas and Perrine Ruby* \\ Lyon Neuroscience Research Center, Brain Dynamics and Cognition Team (DYCOG), INSERM UMRS 1028, CNRS UMR \\ 5292, Université Claude Bernard Lyon 1, Université de Lyon, Lyon, France
}

OPEN ACCESS

Edited by: Caroline L. Horton, Bishop Grosseteste University, United Kingdom

Reviewed by:

Roumen Kirov, Institute of Neurobiology (BAS), Bulgaria

Elizaveta Solomonova Université de Montréal, Canada

${ }^{*}$ Correspondence: Perrine Ruby perrine.ruby@inserm.fr

Specialty section: This article was submitted to Cognitive Science, a section of the journal Frontiers in Psychology

Received: 27 July 2018 Accepted: 11 September 2018 Published: 27 September 2018

Citation:

Vallat $R$, Eichenlaub J-B, Nicolas $A$ and Ruby P (2018) Dream Recall Frequency Is Associated With Medial Prefrontal Cortex White-Matter Density. Front. Psychol. 9:1856. doi: 10.3389/fpsyg.2018.01856
Recent findings indicate that dream recall frequency (DRF) is associated with neurophysiological traits, and notably the regional cerebral blood flow at rest within the medial prefrontal cortex (MPFC) and the temporo-parietal junction (TPJ). To test whether, such physiological traits are rooted in anatomical specificities, we used voxel-based morphometry to compare the white matter and gray matter density in regions related to dream recall (either at the experimental or theoretical level, MPFC, TPJ, hippocampus and amygdala) between 46 high dream recallers (HR, DRF $=5.98 \pm 1.25$ days per week with a dream report) and 46 low dream recallers ( $L R, D R F=0.34 \pm 0.29)$. We found an increased medial prefrontal cortex white-matter density in HR compared to LR but no other significant difference between the two groups. These results are consistent with previous studies showing that lesions within the white matter of medial prefrontal cortex are associated with a partial or total cessation of dream reporting and suggest an implication of this region in dream recall or, more likely, in dream production.

Keywords: dreaming, dream recall, voxel-based morphometry, sleep, MRI, hippocampus, amygdala, DMN

\section{INTRODUCTION}

While dreaming has long been considered as the cognitive correlate of rapid eye movement (REM) sleep, it is now well established that dreaming can occur in any sleep stage and is therefore not exclusive to a specific sleep stage (Solms, 2000; Nir and Tononi, 2010; Ruby, 2011; Montangero, 2018). It is consequently currently out of reach to know for sure when one is actually dreaming while asleep (i.e., no physiological marker of dreaming has been discovered yet). For that reason, most empirical investigation of dreaming (be it the investigation of dream content, or frequency) are based on the study of dream memories reported after the awakening of the dreamer (e.g., Schwartz and Maquet, 2002; Fosse et al., 2003; Schredl et al., 2003; Schwartz, 2003; Zadra and Robert, 2012; Windt, 2013; Vallat et al., 2017a). Studies on dreaming have thus highlighted the cognitive and cerebral correlates of dream recall, either by investigating the EEG in the minutes preceding an awakening followed (or not) by a dream recall (Esposito et al., 2004; Wittmann et al., 2004; Chellappa et al., 2011; Marzano et al., 2011; Scarpelli et al., 2015; Siclari et al., 2017), or by

Abbreviations: BMI, body mass index; DMN, default mode network; DRF, dream recall frequency; EEG, electroencephalography; fMRI, functional magnetic resonance imaging; HR, high dream recallers; LR, low dream recallers; MPFC, medial prefrontal cortex; PET, positron emission tomography; rCBF, regional cerebral blood flow; TPJ, temporoparietal junction; VBM, voxel-based morphometry. 
evaluating the cognitive and brain functioning associated with high and low DRF using behavioral methods, EEG or fMRI (Ruby, 2011 for a review; Eichenlaub et al., 2014a,b; Vallat et al., 2017b, 2018a).

Using this last strategy, recent works from our team highlighted several neurophysiological differences between HR (more than 3 days per week with a dream recall) and LR (less than 2 days per month with a dream recall), not only during sleep but also during wakefulness (Eichenlaub et al., 2014a,b; Vallat et al., 2017b). Notably, using PET, we compared the spontaneous rCBF of HR and LR during sleep and wakefulness, and showed that HR have a higher spontaneous $\mathrm{CCBF}$ than LR in the temporo-parietal junction (TPJ) and in the MPFC during REM sleep, N3 sleep and wakefulness (Eichenlaub et al., 2014b).

We concluded that these two regions played a key role in dream production or recall since lesions of these same areas have been found to be consistently associated with global or partial cessation of dream reporting (without any concurrent sleep disturbance; see Solms, 1997, 2000).

To our knowledge, only one study has so far investigated the relationship between brain anatomy and dream recall (De Gennaro et al., 2011). In this study, the authors used multiple regression analysis to evaluate the linear relationships between measures of some deep gray matter structures (amygdala and hippocampus) and quantitative and qualitative aspects of dream reports. They reported that the neuroanatomical measures were not associated with the number of dreams recalled per day, but they were with some features of dream reports such as length, emotional load, bizarreness, and vividness.

In the present study, we intended to further test the possible association between brain anatomical structures and dream report frequency. In line with previous results, we targeted brain regions previously associated with dream recall at the experimental or theoretical level (MPFC \& TPJ, Solms, 1997; Eichenlaub et al., 2014b; hippocampus and amygdala, Maquet et al., 2005; Levin and Nielsen, 2007; De Gennaro et al., 2011; Perogamvros and Schwartz, 2012; Llewellyn, 2013; Malinowski and Horton, 2015). We expected that HR would show an increased density of the gray-matter and/or white-matter in the MPFC and/or the TPJ but would not show such an increase in the hippocampus and the amygdala.

\section{METHODS}

\section{Participants}

Data for this study comes from two distinct neuroimaging studies (Eichenlaub et al., 2014b; Vallat et al., 2018b). In both studies, the main inclusion criterion was self-reported habitual weekly DRF, assessed by questionnaires (Vallat et al., 2018a). The inclusion criteria for HR were at least 3 days per week with a dream recall, and for LR, at most 2 days per month with a dream recall. No subjects had a history of medical, neurological, or psychiatric disease or was on medication at the time of the studies. The subjects provided written informed consent according to the Declaration of Helsinki. The studies were approved by the local ethics committee (CCPPRB, Centre Leon Berard, Lyon, France), and the subjects were paid for participation.

The anatomical scans acquired during wakefulness of 92 participants were analyzed (mean age $=22.45$, standard deviation $=2.23$, range $=19-29)$. Among them, 46 were HR and 46 were LR. As can be seen in Table 1, the two groups differed significantly in DRF, but not in age, sex ratio and body mass index (BMI).

\section{MRI Data Collection}

Structural data for both studies were acquired at the CERMEP neuroimaging facility in Lyon, France. In Study A (21 HR and 20 LR) (Eichenlaub et al., 2014b), structural MRI were acquired with a T1-weighted MPRAGE sequence (1.0 mm isotropic resolution) on a $1.5 \mathrm{~T}$ Siemens Sonata MR system (Siemens Medical Solutions, Erlangen, Germany). In Study B, structural MRI were acquired with a T1-weighted MPRAGE sequence (0.9 $\mathrm{mm}$ isotropic resolution) on a $3 \mathrm{~T}$ Siemens Primage MR system (28 HR and 27 LR, Vallat et al., 2018b). Out of the 96 subjects, four were excluded based on age or BMI outlier rejection.

\section{VBM Analyses Preprocessing}

Prior to preprocessing, all raw images were visually inspected for potential artifacts. Data preprocessing was performed using the CAT12 toolbox $^{1}$ (Gaser and Dahnke, 2016) for SPM12 (Wellcome Department of Cognitive Neurology). Data were normalized to MNI stereotactic space using DARTEL registration (Ashburner, 2007), corrected for bias field inhomogeneities, and segmented into gray matter, white matter, and cerebrospinal fluid. The mean correlation and weighted overall image quality were then computed and visually plotted to perform quality check and outlier rejection. The mean correlation measures the homogeneity of the data after pre-processing, whereas the weighted overall image quality combines measurements of noise and spatial resolution of the image before preprocessing. Four participants were excluded from further analyses because of a low data quality (one HR and one LR from Study A, one HR and one LR from study B). For the remaining 88 participants, we estimated the total intracranial volume (TIV) to further correct for different head size and volume. Images were then smoothed with an isotropic Gaussian kernel of $6 \mathrm{~mm}$ full width at halfmaximum.

$\overline{{ }^{1} \text { http://www.neuro.uni-jena.de/cat/ }}$

TABLE 1 | Group demographics. P-values were obtained using two-sided $t$-tests for age, DRF, and BMl and using chi-square for the sex ratio.

\begin{tabular}{lccccc}
\hline & $\boldsymbol{n}$ & Age & Sex ratio (M/F) & Habitual Weekly DRF & BMI \\
\hline Mean HR & 46 & 22.52 & 2.1 & 5.98 & 22.63 \\
STD HR & - & 2.22 & - & 1.25 & 2.36 \\
Mean LR & 46 & 22.38 & 2.8 & 0.34 & 22.45 \\
STD LR & - & 2.26 & - & 0.29 & 2.50 \\
p-value & - & 0.77 & 0.49 & $<0.001$ & 0.71
\end{tabular}




\section{Statistical Design}

The normalized modulated smoothed white and gray matter images were entered into an independent two sample $T$-test statistical model, together with age and TIV as covariates. We restricted the analyses to the MPFC, TPJ, hippocampus and amygdala (Supplementary Figure S1). A single binary spatial mask comprising these four regions was created by combining the individual and thresholded spatial masks of each of these regions (using the fslmaths command). The individual spatial masks were defined by generating a map in Neurosynth ${ }^{2}$, using the keywords 'amygdala,' 'hippocampus,' 'medial prefrontal', and 'temporoparietal junction,' respectively. The four maps were then thresholded at $z=7$ to increase anatomical specificity. The significance threshold was set at $p<0.001$ uncorrected with an extent threshold of 10 voxels.

\section{RESULTS}

We did not find gray matter density differences between HR and LR. However, VBM analyses revealed an increased MPFC white-matter density in HR compared to LR (peak cluster in MNI coordinates $=3,56,11$; extent $=172$ voxels; Figure 1).

\section{DISCUSSION}

This study intended to test whether an increased DRF in HR could be associated with gray- or white- matter density specificities in brain regions previously associated (at the theoretical or experimental level) with dream recall and/or production, namely the amygdala, hippocampus, MPFC and TPJ. VBM analyses of the anatomical scans of $44 \mathrm{HR}$ and $44 \mathrm{LR}$ revealed a significant difference between the two groups in the white matter of the MPFC. This result adds an anatomical dimension to numerous experimental findings showing differences in brain functioning between $\mathrm{HR}$ and LR (Eichenlaub et al., $2014 a, b)$. To the best of our knowledge, this is the first

${ }^{2}$ http://neurosynth.org/ study reporting brain structural differences between HR and LR.

The absence of a significant group difference in the amygdala and hippocampus replicate previous results (Torda, 1969; De Gennaro et al., 2011) and support the idea that these two regions are not directly involved in dream memory frequency, even if they might be involved in some qualitative aspects of dream content (Torda, 1969; Maquet and Franck, 1997; Revonsuo, 2000; Maquet et al., 2005; Nielsen and Stenstrom, 2005; De Gennaro et al., 2011; Solms, 2013; Corsi-Cabrera et al., 2016).

The significant group difference in the white-matter of the MPFC is well in line with previous neuropsychological findings showing a cessation of dream reports after lesion in the white matter surrounding the frontal horns of the lateral ventricles (Solms, 1997, 2000). To explain this observation, Solms stressed that the ventromedial prefrontal cortex contains a substantial number of fibers connecting frontal and limbic structures with dopaminergic cells in the ventral tegmentum. $\mathrm{He}$ further suggested that dreaming is generated by this dopamine circuit, i.e., that the mesocortical-mesolimbic dopamine system plays a causal role in the generation of dreams (Solms, 2000). Our results support this conclusion by showing in healthy subjects a significant association between DRF and the white matter density in the MPFC. To assess whether it is an acquired or an innate characteristic, and to better understand the functional significance of a white matter density increase, future studies may measure the white matter density in the MPFC before and after an increase of DRF induced by an increase in attention to, or interest in dreams (as can be done with a dream diary, Schredl, 2002; Ruby, 2011).

At the functional level, some EEG studies have produced results compatible with an involvement of MPFC in dream recall (e.g., a positive association between increased frontal theta EEG power and successful dream recall, Marzano et al., 2011; Scarpelli et al., 2015) and a PET study has demonstrated an increased cerebral blood flow in the MPFC during sleep in HR as compared to LR (Eichenlaub et al., 2014b). This last result suggests that MPFC plays a role in dream production rather than in dream recall due to its tonic activity during sleep. In addition, previous results did not noticeably involve MPFC in memory

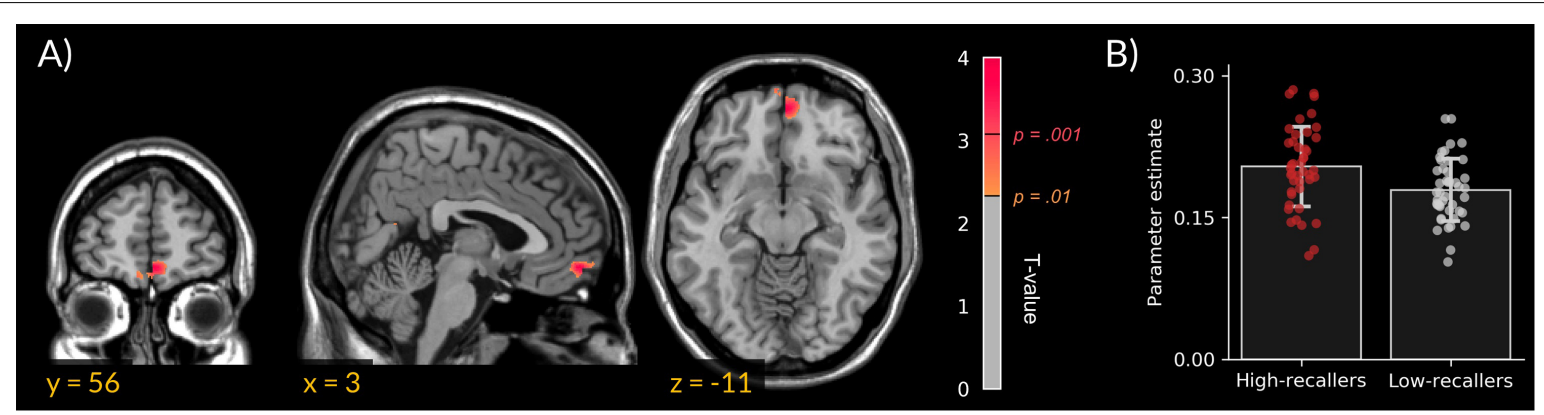

FIGURE 1 | Increased medial prefrontal cortex white-matter density in high dream recallers. (A) Statistical parametric map showing an increased white-matter density in HR $(N=44)$ as compared to LR $(N=44$; peak cluster in MNI coordinates $=3,56,-11)$. For display purpose, threshold was set at $p<0.01$ uncorrected. (B) Bar plots of cluster-averaged parameter estimate in both groups. Points represent individual values. 
recall. Rather, this region is involved in social cognition such as mind reading (Legrand and Ruby, 2009 for a review), social emotions processing (e.g., Ruby and Decety, 2004) and projective imagery (i.e., envisioning the future or the past, Buckner and Carroll, 2007). As such, it could participate in the production of the scenario or plot of the dream. This is consistent with results showing a similar level of $\mathrm{rCBF}$ in the MPFC during wake and REM sleep and an important drop of activity in this region during N3 sleep, a sleep stage associated with less dream reports than REM sleep (Nielsen, 2000; Maquet et al., 2005; Ruby, 2011). This interpretation is well in line with recent review articles arguing that dreaming is an intensified form of mind wandering and that at least part of the DMN of the brain (of which the MPFC is a core component, Gusnard and Raichle, 2001; Raichle et al., 2001) is involved in the production of dreams (Domhoff, 2011; Domhoff and Fox, 2015; Christoff et al., 2016). The DMN is a set of functionally coupled brain regions especially during internally oriented mental processes and episodic memory retrieval (Gusnard and Raichle, 2001; Raichle et al., 2001; Legrand and Ruby, 2009). This network is centered on the MPFC, the posterior cingulate cortex (PCC) and the lateral parietal areas around the TPJ area but also comprises the temporal pole and the hippocampus and parahippocampal gyrus (Legrand and Ruby, 2009; Raichle, 2015).

Finally, it should be noted that in this study, we investigated the possible brain anatomical correlates of the trait component of DRF. Yet, DRF has both trait and state components (Schredl and Reinhard, 2008; Ruby, 2011; Eichenlaub et al., 2014a,b). The state components of DRF mediated by, for example, sleep stage, time of night, the pre-sleep mood, or psychotropic drug use (Stickgold et al., 2001; Schredl, 2007; Nir and Tononi, 2010; Ruby, 2011; Tribl et al., 2013) most probably has functional rather than anatomical neurophysiological correlates (e.g., Esposito et al., 2004; Chellappa et al., 2011; Marzano et al., 2011; Scarpelli et al., 2015).

The main finding of this article is that, as compared to LR, HR show an increased white matter density in the MPFC. Altogether

\section{REFERENCES}

Ashburner, J. (2007). A fast diffeomorphic image registration algorithm. Neuroimage 38, 95-113. doi: 10.1016/j.neuroimage.2007.07.007

Buckner, R. L., and Carroll, D. C. (2007). Self-projection and the brain. Trends Cogn. Sci. 11, 49-57. doi: 10.1016/j.tics.2006.11.004

Chellappa, S. L., Frey, S., Knoblauch, V., and Cajochen, C. (2011). Cortical activation patterns herald successful dream recall after NREM and REM sleep. Biol. Psychol. 87, 251-256. doi: 10.1016/j.biopsycho.2011.0 3.004

Christoff, K., Irving, Z. C., Fox, K. C. R., Spreng, R. N., and Andrews-Hanna, J. R. (2016). Mind-wandering as spontaneous thought: a dynamic framework. Nat. Rev. Neurosci. 17, 718-731. doi: 10.1038/nrn.2016.113

Corsi-Cabrera, M., Velasco, F., del Río-Portilla, Y., Armony, J. L., TrejoMartínez, D., Guevara, M. A., et al. (2016). Human amygdala activation during rapid eye movements of rapid eye movement sleep: an intracranial study. J. Sleep Res. 25, 576-582. doi: 10.1111/jsr.12415

De Gennaro, L., Cipolli, C., Cherubini, A., Assogna, F., Cacciari, C., Marzano, C., et al. (2011). Amygdala and hippocampus volumetry and diffusivity in relation to dreaming. Hum. Brain Mapp. 32, 1458-1470. doi: 10.1002/hbm.21120 with previous functional (PET, EEG) and neuropsychological (lesions) results, our finding argues for a role of the MPFC in dream production.

\section{AUTHOR CONTRIBUTIONS}

$\mathrm{AN}, \mathrm{J}-\mathrm{BE}, \mathrm{RV}$, and PR participated in designing the study and collecting data. RV conducted the data analysis and wrote the first draft of the paper. PR participated in the writing of the article.

\section{FUNDING}

This research was funded by the CRNL and conducted in the framework of the LabEx Cortex (ANR-11-LABX-0042) of Université de Lyon, within the program "Investissements d'avenir" (ANR-11-IDEX-0007) operated by the French National Research Agency (ANR).

\section{ACKNOWLEDGMENTS}

The authors would like to thank Basak Turker, Morgane Hamon, Franck Lamberton, and Danielle Ibarrola for substantial help in data collection and analysis, as well as Jamila Lagha for her help in administrative work.

\section{SUPPLEMENTARY MATERIAL}

The Supplementary Material for this article can be found online at: https://www.frontiersin.org/articles/10.3389/fpsyg. 2018.01856/full\#supplementary-material

FIGURE S1 | Spatial mask used in the VBM analysis. MPFC, medial prefrontal cortex. TPJ, temporoparietal junction.

Domhoff, G. W. (2011). The neural substrate for dreaming: is it a subsystem of the default network? Conscious. Cogn. 20, 1163-1174. doi: 10.1016/j.concog.2011. 03.001

Domhoff, G. W., and Fox, K. C. R. (2015). Dreaming and the default network: a review, synthesis, and counterintuitive research proposal. Conscious. Cogn. 33, 342-353. doi: 10.1016/j.concog.2015.01.019

Eichenlaub, J.-B., Bertrand, O., Morlet, D., and Ruby, P. (2014a). Brain reactivity differentiates subjects with high and low dream recall frequencies during both sleep and wakefulness. Cereb. Cortex 24, 1206-1215. doi: 10.1093/cercor/bhs388

Eichenlaub, J.-B., Nicolas, A., Daltrozzo, J., Redouté, J., Costes, N., and Ruby, P. (2014b). Resting brain activity varies with dream recall frequency between subjects. Neuropsychopharmacology 39, 1594-1602. doi: 10.1038/npp.2014.6

Esposito, M. J., Nielsen, T. A., and Paquette, T. (2004). Reduced Alpha power associated with the recall of mentation from Stage 2 and Stage REM sleep. Psychophysiology 41, 288-297. doi: 10.1111/j.1469-8986.00143.x

Fosse, M. J., Fosse, R., Hobson, J. A., and Stickgold, R. (2003). Dreaming and episodic memory: a functional dissociation? J. Cogn. Neurosci. 15, 1-9. doi: 10.1162/089892903321107774

Gaser, C., and Dahnke, R. (2016). CAT-a computational anatomy toolbox for the analysis of structural MRI data. HBM 2016, 336-348. 
Gusnard, D. A., and Raichle, M. E. (2001). Searching for a baseline: functional imaging and the resting human brain. Nat. Rev. Neurosci. 2, 685-694. doi: $10.1038 / 35094500$

Legrand, D., and Ruby, P. (2009). What is self-specific? Theoretical investigation and critical review of neuroimaging results. Psychol. Rev. 116, 252-282. doi: $10.1037 / \mathrm{a} 0014172$

Levin, R., and Nielsen, T. A. (2007). Disturbed dreaming, posttraumatic stress disorder, and affect distress: a review and neurocognitive model. Psychol. Bull. 133, 482-528. doi: 10.1037/0033-2909.133.3.482

Llewellyn, S. (2013). Such stuff as dreams are made on? Elaborative encoding, the ancient art of memory, and the hippocampus. Behav. Brain Sci. 36, 589-607. doi: 10.1017/S0140525X12003135

Malinowski, J. E., and Horton, C. L. (2015). Metaphor and hyperassociativity: the imagination mechanisms behind emotion assimilation in sleep and dreaming. Front. Psychol. 6:1132. doi: 10.3389/fpsyg.2015.01132

Maquet, P., and Franck, G. (1997). REM sleep and amygdala. Mol. Psychiatry 2, 195-196. doi: 10.1038/sj.mp.4000239

Maquet, P., Ruby, P., Maudoux, A., Albouy, G., Sterpenich, V., Dang-Vu, T. T., et al. (2005). Human cognition during REM sleep and the activity profile within frontal and parietal cortices: a reappraisal of functional neuroimaging data. Prog. Brain Res. 150, 219-595. doi: 10.1016/S0079-6123(05)50 016-5

Marzano, C., Ferrara, M., Mauro, F., Moroni, F., Gorgoni, M., Tempesta, D., et al. (2011). Recalling and forgetting dreams: theta and alpha oscillations during sleep predict subsequent dream recall. J. Neurosci. 31, 6674-6683. doi: 10.1523/ JNEUROSCI.0412-11.2011

Montangero, J. (2018). Dreaming and REM sleep: history of a scientific denial whose disappearance entailed a reconciliation of the neuroscience and the cognitive psychological approaches to dreaming. Int. J. Dream Res. 11, 30-45.

Nielsen, T. A. (2000). A review of mentation in REM and NREM sleep: "covert" REM sleep as a possible reconciliation of two opposing models. Behav. Brain Sci. 23, 851-866. doi: 10.1017/S0140525X0000399X

Nielsen, T. A., and Stenstrom, P. (2005). What are the memory sources of dreaming? Nature 437, 1286-1289. doi: 10.1038/nature04288

Nir, Y., and Tononi, G. (2010). Dreaming and the brain: from phenomenology to neurophysiology. Trends Cogn. Sci. 14, 88-100. doi: 10.1016/j.tics.2009.12.001

Perogamvros, L., and Schwartz, S. (2012). The roles of the reward system in sleep and dreaming. Neurosci. Biobehav. Rev. 36, 1934-1951. doi: 10.1016/j. neubiorev.2012.05.010

Raichle, M. E. (2015). The brain's default mode network. Annu. Rev. Neurosci. 38, 433-447. doi: 10.1146/annurev-neuro-071013-014030

Raichle, M. E., MacLeod, A. M., Snyder, A. Z., Powers, W. J., Gusnard, D. A., and Shulman, G. L. (2001). A default mode of brain function. Proc. Natl. Acad. Sci. U.S.A. 98, 676-682. doi: 10.1073/pnas.98.2.676

Revonsuo, A. (2000). The reinterpretation of dreams: an evolutionary hypothesis of the function of dreaming. Behav. Brain Sci. 23, 877-901. doi: 10.1017/ S0140525X00004015

Ruby, P. (2011). Experimental research on dreaming: state of the art and neuropsychoanalytic perspectives. Front. Psychol. 2:286. doi: 10.3389/fpsyg. 2011.00286

Ruby, P., and Decety, J. (2004). How would you feel versus how do you think she would feel? A neuroimaging study of perspective-taking with social emotions. J. Cogn. Neurosci. 16, 988-999. doi: 10.1162/0898929041502661

Scarpelli, S., Marzano, C., D’Atri, A., Gorgoni, M., Ferrara, M., and De Gennaro, L. (2015). State- or trait-like individual differences in dream recall: preliminary findings from a within-subjects study of multiple nap REM sleep awakenings. Front. Psychol. 6:928. doi: 10.3389/fpsyg.2015.00928

Schredl, M. (2002). Questionnaires and diaries as research instruments in dream research: methodological issues. Dreaming 12, 17-26. doi: 10.1023/A: 1013890421674

Schredl, M. (2007). "Dream recall: models and empirical data," in The New Science of Dreaming - Volume II. Content, Recall and Personality Correlates of Dreams, eds D. Barrett and P. Mc Namara (Westport, CT: Praeger Publishing).
Schredl, M., and Reinhard, I. (2008). Dream recall, dream length, and sleep duration: state or trait factor. Percept. Mot. Skills 106, 633-636. doi: 10.2466/ pms.106.2.633-636

Schredl, M., Wittmann, L., Ciric, P., and Götz, S. (2003). Factors of home dream recall: a structural equation model. J. Sleep Res. 12, 133-141. doi: 10.1046/j. 1365-2869.2003.00344.x

Schwartz, S. (2003). Are life episodes replayed during dreaming? Trends Cogn. Sci. 7, 325-327.

Schwartz, S., and Maquet, P. (2002). Sleep imaging and the neuro-psychological assessment of dreams. Trends Cogn. Sci. 6, 23-30. doi: 10.1016/S1364-6613(00) 01818-0

Siclari, F., Baird, B., Perogamvros, L., Bernardi, G., LaRocque, J. J., Riedner, B., et al. (2017). The neural correlates of dreaming. Nat. Neurosci. 20, 872-878. doi: $10.1038 / \mathrm{nn} .4545$

Solms, M. (1997). The Neuropsychology of Dreams: A Clinico-Anatomical Study. Mahwah: L. Erlbaum.

Solms, M. (2000). Dreaming and REM sleep are controlled by different brain mechanisms. Behav. Brain Sci. 23, 843-850. doi: 10.1017/S0140525X00003988

Solms, M. (2013). Dreaming is not controlled by hippocampal mechanisms. Behav. Brain Sci. 36, 629; discussion 634-59. doi: 10.1017/S0140525X1300143X

Stickgold, R., Malia, A., Fosse, R., Propper, R., and Hobson, J. A. (2001). Brainmind states: I. Longitudinal field study of sleep/wake factors influencing mentation report length. Sleep. 24, 171-179. doi: 10.1093/sleep/24.2.171

Torda, C. (1969). Dreams of subjects with bilateral hippocampal lesions. Acta Psychiatr. Scand. 45, 277-288. doi: 10.1111/j.1600-0447.1969.tb07128.x

Tribl, G. G., Wetter, T. C., and Schredl, M. (2013). Dreaming under antidepressants: a systematic review on evidence in depressive patients and healthy volunteers. Sleep Med. Rev. 17, 133-142. doi: 10.1016/j.smrv.2012. 05.001

Vallat, R., Chatard, B., Blagrove, M., and Ruby, P. (2017a). Characteristics of the memory sources of dreams: a new version of the content-matching paradigm to take mundane and remote memories into account. PLoS One 12:e0185262. doi: 10.1371/journal.pone.0185262

Vallat, R., Lajnef, T., Eichenlaub, J.-B., Berthomier, C., Jerbi, K., Morlet, D., et al. (2017b). Increased evoked potentials to arousing auditory stimuli during sleep: implication for the understanding of dream recall. Front. Hum. Neurosci. 11:132. doi: 10.3389/fnhum.2017.00132

Vallat, R., Eskinazi, M., Nicolas, A., and Ruby, P. (2018a). Sleep and dream habits in a sample of French college students who report no sleep disorders. J. Sleep Res. 27:e12659. doi: 10.1111/jsr.12659

Vallat, R., Meunier, D., Nicolas, A., and Ruby, P. (2018b). Hard to wake up? The cerebral correlates of sleep inertia assessed using combined behavioral, EEG and fMRI measures. Neuroimage 184, 266-278. doi: 10.1016/j.neuroimage.2018.09. 033

Windt, J. M. (2013). Reporting dream experience: why (not) to be skeptical about dream reports. Front. Hum. Neurosci. 7:708. doi: 10.3389/fnhum.2013.00708

Wittmann, L., Palmy, C., and Schredl, M. (2004). NREM sleep dream recall, dream report length and cortical activation. Sleep Hypn. 6, 54-58.

Zadra, A., and Robert, G. (2012). Dream recall frequency: impact of prospective measures and motivational factors. Conscious. Cogn. 21, 1695-1702. doi: 10. 1016/j.concog.2012.08.011

Conflict of Interest Statement: The authors declare that the research was conducted in the absence of any commercial or financial relationships that could be construed as a potential conflict of interest.

Copyright (C) 2018 Vallat, Eichenlaub, Nicolas and Ruby. This is an open-access article distributed under the terms of the Creative Commons Attribution License (CC BY). The use, distribution or reproduction in other forums is permitted, provided the original author(s) and the copyright owner(s) are credited and that the original publication in this journal is cited, in accordance with accepted academic practice. No use, distribution or reproduction is permitted which does not comply with these terms. 